\title{
Comparison between heparin-conjugated fibrin and collagen sponge as bone morphogenetic protein-2 carriers for bone regeneration
}

\author{
Hee Seok Yang ${ }^{1 *}$, Wan-Geun La ${ }^{1 *}$, \\ Yong-Min $\mathrm{Cho}^{1}$, Wangsoo Shin ${ }^{2}$, Guw-Dong $\mathrm{Yeo}^{2}$ \\ and Byung-Soo Kim ${ }^{1,3,4}$ \\ ${ }^{1}$ School of Chemical and Biological Engineering \\ Seoul National University \\ Seoul 151-744, Korea \\ ${ }^{2}$ Samyang Corporation Pharmaceutical R\&D Center \\ Daejeon 305-717, Korea \\ ${ }^{3}$ Bio-Max Institute \\ Institute of Chemical Process \\ Engineering Research Institute \\ Seoul National University \\ Seoul 151-744, Korea \\ ${ }^{4}$ Corresponding author: Tel, 82-2-880-1509; \\ Fax, 82-2-880-1604; E-mail, byungskim@ @snu.ac.kr \\ *These authors contributed equally to this work. \\ http://dx.doi.org/10.3858/emm.2012.44.5.039
}

Accepted 6 February 2012

Available Online 10 February 2012

Abbreviations: ALP, alkaline phosphatase; BMP-2, bone morphogenetic protein-2; FDA, Food and Drug Administration; HCF, heparin-conjugated fibrin; micro-CT, microcomputed tomography

\begin{abstract}
Bone morphogenetic protein-2 (BMP-2) is used to promote bone regeneration. However, the bone regeneration ability of BMP-2 relies heavily on the delivery vehicle. Previously, we have developed heparin-conjugated fibrin (HCF), a vehicle for long-term delivery of BMP-2 and demonstrated that long-term delivery of BMP-2 enhanced its osteogenic efficacy as compared to short-term delivery at an equivalent dose. The aim of this study was to compare the bone-forming ability of the BMP-2 delivered by HCF to that delivered by clinically utilized BMP-2 delivery vehicle collagen sponge. An in vitro release profile of BMP-2 showed that $\mathrm{HCF}$ released $80 \%$ of the loaded BMP-2 within 20
\end{abstract}

days, whereas collagen sponge released the same amount within the first 6 days. Moreover, the BMP-2 released from the HCF showed significantly higher alkaline phosphatase activity than the BMP-2 released from collagen sponge at 2 weeks in vitro. Various doses of BMP-2 were delivered with HCF or collagen sponge to mouse calvarial defects. Eight weeks after the treatment, bone regeneration was evaluated by computed tomography, histology, and histomorphometric analysis. The dose of BMP-2 delivered by HCF to achieve $100 \%$ bone formation in the defects was less than half of the BMP-2 dose delivered by collagen sponge to achieve a similar level of bone formation. Additionally, bone regenerated by the HCF-BMP-2 had higher bone density than bone regenerated by the collagen sponge-BMP-2. These data demonstrate that HCF as a BMP-2 delivery vehicle exerts better osteogenic ability of BMP-2 than collagen sponge, a clinically utilized delivery vehicle.

Keywords: bone morphogenetic protein 2; collagen; drug delivery systems; fibrin; osteogenesis

\section{Introduction}

The application of bone morphogenetic proteins (BMPs) has recently emerged as an effective treatment in bone reconstruction surgery (Friedlaender, 2004; Termaat et al., 2005). BMPs induce bone formation by regulating the recruitment and differentiation of osteoprogenitor cells (Derner and Anderson, 2005; Bessa et al., 2008; Kanakaris and Giannoudis, 2008). Among BMPs, BMP-2 and BMP-7 are now being used clinically (Mont et al., 2004). However, there are several limitations in the clinical application of BMPs. Firstly; BMP-2 may lose its bioactivity due to its short half-life (Takahashi et al., 2005). Additionally, a large dose of BMP-2 is required for clinical treatment due to its rapid loss through diffusion (Carter et al., 2008). Such limitations require large doses of BMP-2 to be used clinically, resulting in high treatment costs and undesirable side effects including bone over- 
growth and immune responses (Shields et al., 2006).

To overcome these problems, it is necessary to develop an appropriate delivery vehicle that can release BMP-2 locally over an extended period and at sufficient concentrations (Issa et al., 2008; La et al., 2010). The osteogenic efficacy, bioactivity, and optimal dosage of BMP-2 are dependent on its delivery vehicle (Issa et al., 2008; La et al., 2010; Yang et al., 2010). Our previous study has demonstrated that long-term delivery vehicles of BMP-2 enhance osteogenic efficacy of the protein compared with short-term delivery vehicles at an equivalent dose (Jeon et al., 2008; Yang et al., 2010). Long-term delivery vehicles enable sustained release and retain the bioactivity of BMP-2 (Jeon et al., 2008). Additionally, bone formation by BMP-2 is known to be dose-dependent, and the dose-dependency relies on the BMP-2 delivery vehicle (Patel et al., 2008; La et al., 2010).

A number of vehicles have been designed for the delivery of BMP-2. Among the delivery vehicles, collagen sponge has proven to be effective for therapeutic applications (Liu et al., 2006); thus, it is used clinically as a delivery vehicle for BMP-2 (Liu et al., 2006; Yang et al., 2011). Although the BMP-2-loaded collagen sponge is used clinically, routine BMP-2 application has been hindered by inherent problems. Several studies have demonstrated that the currently used collagen sponges have a large initial burst release and retention of less than $5 \%$ after 14 days of implantation (Uludag et al., 1999; Haidar et al., 2009).

We previously reported that heparin-conjugated fibrin (HCF) worked well as an injectable delivery vehicle for BMP-2 (Yang et al., 2010). The previous results indicated that BMP-2 released from HCF significantly increased alkaline phosphatase (ALP) activity of cultured osteoblasts (Yang et al., 2010). HCF releases BMP-2 over a long-term period,

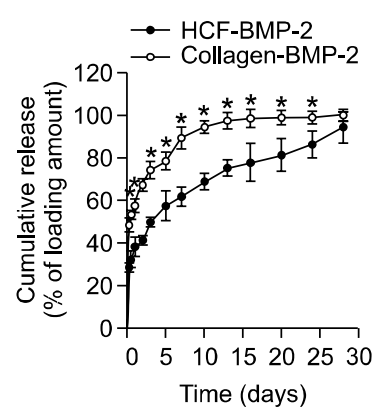

Figure 1. Profiles of BMP-2 release from HCF and collagen sponge. Open circles indicate BMP-2 release from the collagen sponge delivery vehicle, and closed circles indicate BMP-2 release from the HCF delivery vehicle. The values represent the mean \pm standard deviation $(n=8)$. (Collagen: collagen sponge, ${ }^{*} P<0.05$ compared with HCF). while protecting its bioactivity (Yang et al., 2010) because heparin enables BMP-2 release over a long-term period with activity retention through the ionic interactions between heparin and BMP-2 (Zhao et al., 2006). These results suggested that HCF could be utilized to deliver BMP-2 over a long-term period for bone regeneration. The aim of the present study was to compare the efficacy of HCF as a sustained release BMP-2 delivery vehicle to that of collagen sponge, which is the delivery vehicle currently used for clinical purposes of orthotopic bone formation. We hypothesized that bone regeneration relies on the dose of BMP-2 delivered by HCF and that HCF-mediated delivery of BMP-2 enhances bone regeneration compared with collagen sponge-mediated delivery of BMP-2.

\section{Results}

\section{in vitro BMP-2 release}

The profiles of in vitro release of BMP-2 from HCF and collagen sponge were compared (Figure 1). It was observed that the HCF delivery vehicle maintained a sustained release of BMP-2 compared with the collagen sponge vehicle. The amounts of released BMP-2 differed dramatically depending on the delivery vehicles during the first 10 days of BMP-2 release. When compared to the HCF vehicle, the collagen sponge vehicle showed an initial BMP-2 burst during the first 3 days. Approximately $80 \%$ of the BMP-2 loaded on the collagen sponge was released over a period of 6 days, whereas the same dose of BMP-2 was released from the HCF over a period of 20 days.

The ALP activity of cultured rat osteoblasts was significantly higher in the group of BMP-2 released from the HCF than in the group of BMP-2 released

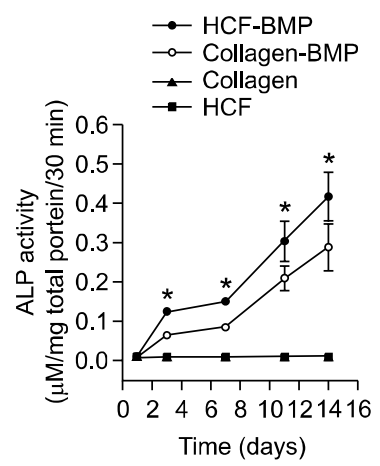

Figure 2. The bioactivity of BMP-2 released from the delivery vehicles, as assessed by measuring ALP activity of rat calvarial osteoblasts cultured with the different delivery systems $\left(n=5,{ }^{*} P<0.05\right.$ between HCF and collagen). 
from the collagen sponge for 14 days (Figure 2). The groups of carriers without BMP-2 showed no ALP activity for 14 days.
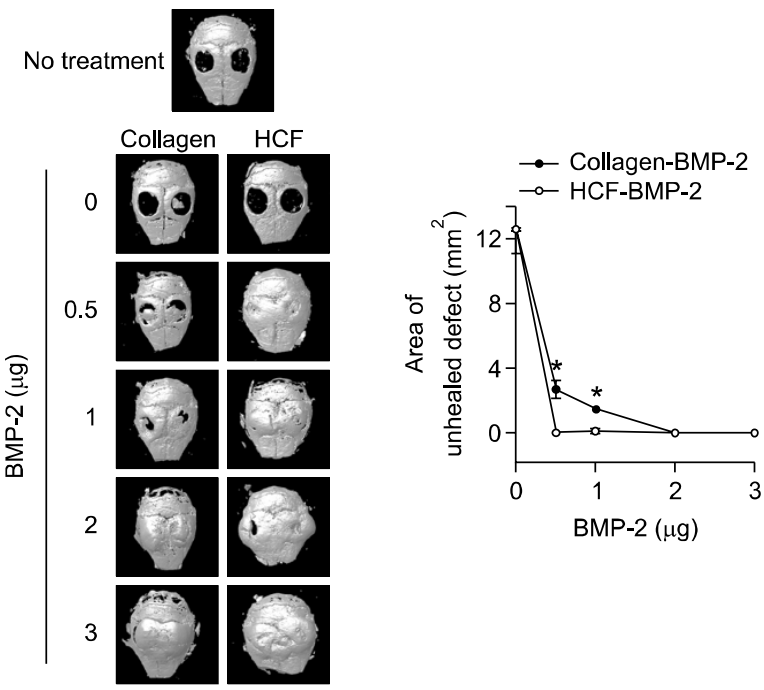

Figure 3. Representative micro-CT images of mouse skulls treated with the different doses of BMP-2 delivered by either collagen or HCF for 8 weeks. Defects were treated with $0.0,0.5,1.0,2.0$, and $3.0 \mu \mathrm{g}$ of BMP-2 delivered with either collagen sponge or HCF vehicle. The area of unhealed defect was determined from the micro-CT images.

\section{Bone formation in vivo}

After 8 weeks of implantation in the mouse calvarial defect models, all samples were analyzed by $\mathrm{X}$-ray microcomputed tomography (micro-CT) examinations (Figure 3 ). The analysis revealed a BMP-2 dose-dependent increase in bone regeneration. The results of the micro-CT evaluations were verified with histological analysis with Goldner's trichrome staining (Figure 4). No bone formation was observed in groups in which BMP-2 treatment was not administered. However, fiber-like tissue was observed in calvarial defect sites (Figure 4). Most of the implanted collagen and HCF resorbed at 8 weeks. Beginning at a BMP-2 dose of $0.5 \mu \mathrm{g}$, however, the HCF delivery vehicle showed more extensive bone formation. Mature bone with lamellar structures and osteocytes in lacunae was observed in groups with BMP-2 doses equal to or higher than $0.5 \mu \mathrm{g}$ in HCF groups. In contrast, the collagen sponge vehicle group showed bone formation to a much lesser extent. Histology revealed excessive areas of bone formation in samples that received high doses of BMP-2. When compared with the collagen sponge delivery vehicle, the HCF delivery vehicle resulted in higher bone density at the same BMP-2 dose.

Bone formation area and bone density were quantified with histomorphometric analysis (Figure $5)$. The group in which no treatment was ad-
A

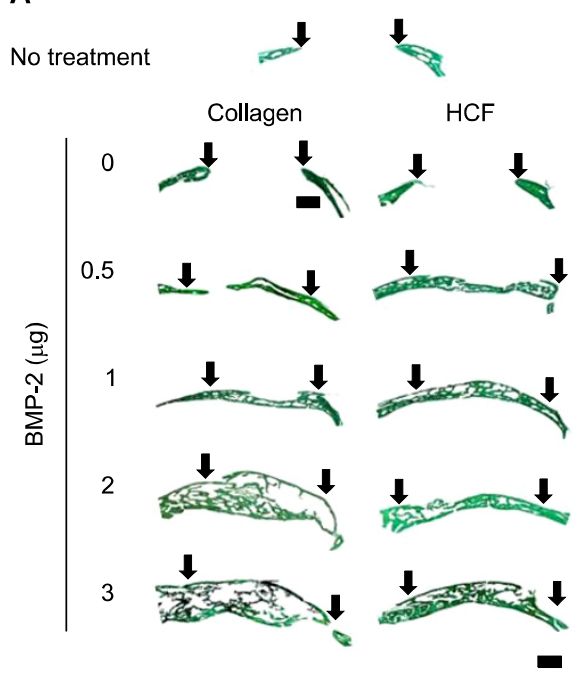

B

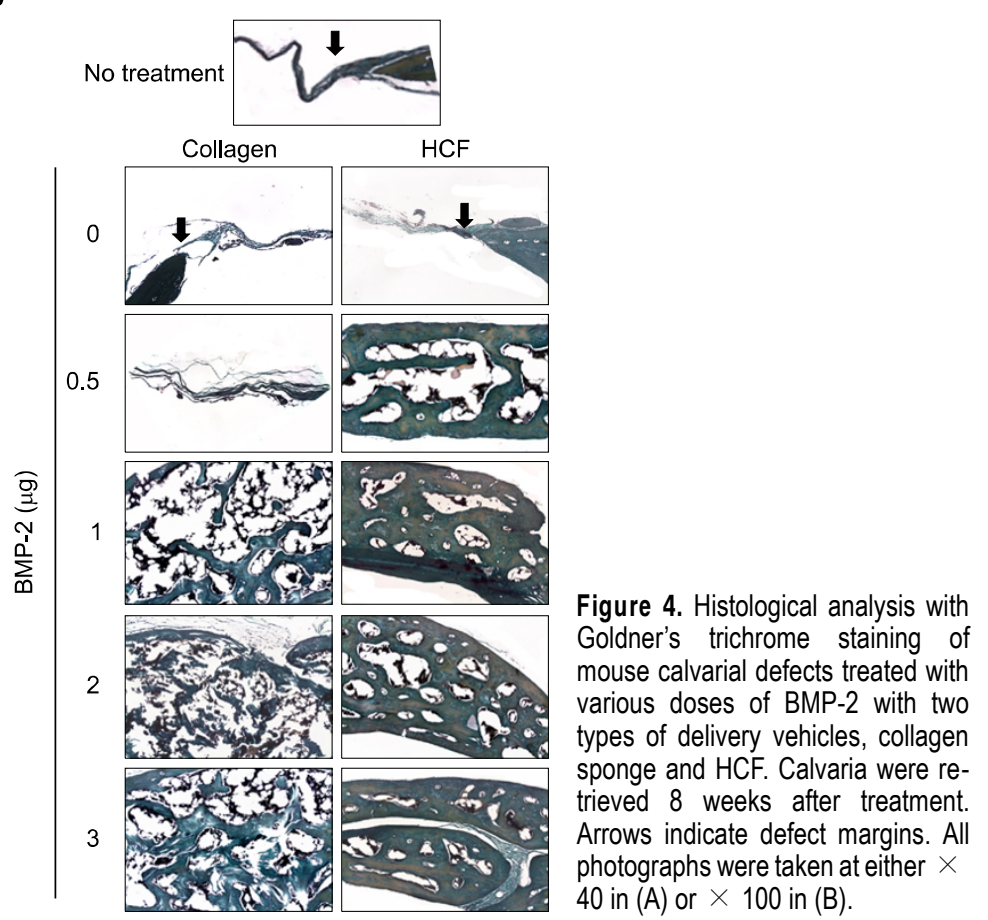



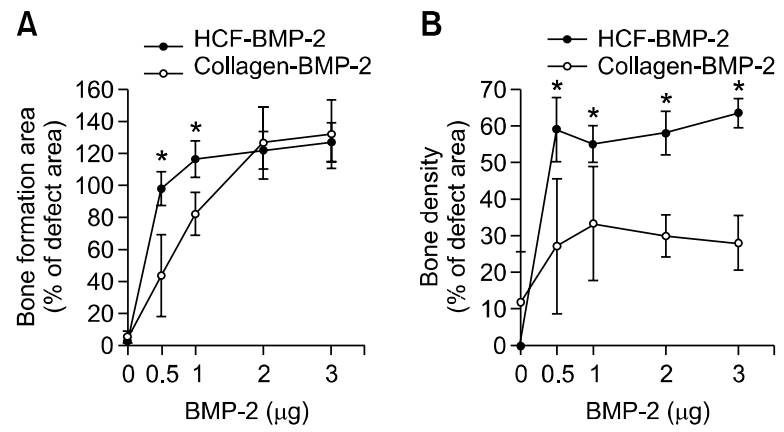

Figure 5. (A) Bone formation area and (B) bone density as determined with histomorphometry analysis $(n=4)$. Open circles indicate BMP-2 delivery with collagen sponge, and closed circles indicate BMP-2 delivery with HCF. The area of bone formation was calculated as bone area/defect area $\times 100\left({ }^{*} P<0.05\right.$ between HCF and collagen), and bone density was calculated as new bone area / (new bone area + fibrous tissue area + residual biomaterials area) $\times 100\left({ }^{*} P<0.05\right.$ between HCF and collagen).

ministered had almost no bone regeneration. In the BMP-2-treated groups, bone regeneration area increased with the BMP-2 dose. At $0.5 \mu \mathrm{g}$ of BMP-2, the bone formation area in the HCF group was nearly $100 \%$ of the defect area (Figure $5 \mathrm{~A}$ ). However, to achieve $100 \%$ of bone regeneration area, the collagen sponge delivery vehicle needed more than $1 \mu \mathrm{g} / \mathrm{defect}$ of BMP-2. The BMP-2 dose required by the HCF delivery vehicle to result in $100 \%$ of the bone regeneration area without excessive bone formation was approximately one-third of that required by the collagen sponge delivery vehicle. Furthermore, the HCF delivery vehicle resulted in significantly higher bone density than the collagen sponge delivery vehicle (Figure 5B). Taken together, micro-CT, histology, and histomorphometric analysis showed that the HCF delivery vehicle for BMP-2 resulted in much more extensive bone formation and higher bone density at lower BMP-2 dosage than the collagen sponge delivery vehicle.

\section{Discussion}

The aim of this study was to compare HCF and collagen sponge, which is a product that is currently used in clinical applications, as BMP-2 delivery vehicles for bone formation. HCF was utilized for sustained delivery of BMP-2 for bone regeneration (Yang et al., 2010). Upon comparing the in vitro release kinetics of BMP-2, the HCF delivery vehicle was superior to the collagen sponge vehicle, more effectively providing a sustained release of BMP-2 (Figure 1). BMP-2 delivery with the HCF vehicle is achieved by electrostatic interactions between the positive charges of the amino acid residues in the BMP-2 protein and the negative charges of the heparin in HCF (Zhao et al., 2006), whereas the collagen sponge relies mostly on adsorption and desorption of BMP-2 protein (Boerckel et al., 2011). These features of HCF allow for the sustained release of BMP-2 protein.

The in vivo bone formation area may depend on the BMP-2 release period (Fu et al., 2008; Issa et al., 2008; Yang et al., 2010). For clinical treatment, high doses of BMP-2 are required because BMP-2 is released rapidly from the delivery vehicle of collagen sponge (Haidar et al., 2009). However, high doses of BMP-2, coupled with a quick release from the delivery vehicle could lead to side effects such as excessive bone formation and immune responses (Shields et al., 2006). These side effects could be reduced by facilitating BMP-2 delivery using HCF. The enhanced bone formation may be a result of not only the sustained delivery of BMP-2 by the HCF, but also the higher ALP activity of the BMP-2 released from the HCF, compared with that released from the collagen sponge (Figure 2).

In addition, bone tissue regenerated with BMP-2 delivery using HCF had a higher bone density than the bone tissue regenerated with BMP-2 delivery using the collagen sponge. This could be due to the different characteristics of HCF and collagen. Tissues other than bone, such as fibrous tissues, could grow easily into collagen sponge because the porous nature of the collagen sponge provides structure and space appropriate for fibrous tissue ingrowth. However, because HCF degrades more quickly than collagen sponge, the ingrowth of fibrous tissue is prevented (Yang et al., 2011).

In conclusion, the present study shows that BMP-2 can be delivered with an HCF vehicle for its sustained release over a sufficiently long period and that HCF as a BMP-2 delivery vehicle resulted in enhanced bone formation compared to the collagen sponge vehicle. In addition, the HCF vehicle used a reduced dose of BMP-2, which was suitable for efficient bone regeneration and could diminish the potential side effects caused by the excessive use of BMP-2. Therefore, the HCF delivery vehicle could improve the current BMP-2 therapy for bone regeneration.

\section{Methods}

\section{Preparation of HCF}

Heparin-conjugated fibrinogen was prepared by covalently bonding heparin (molecular weight $=4,000-6,000$; Sigma, St. 
Louis, MO) to plasminogen-free fibrinogen (Sigma) as previously described (Yang et al., 2010). HCF was formed by mixing heparin-conjugated fibrinogen $(40 \mathrm{mg} / \mathrm{ml})$ and normal fibrinogen $(60 \mathrm{mg} / \mathrm{ml})$ with factor XIII, aprotinin (100 $\mathrm{KIU} / \mathrm{ml})$, calcium chloride $(6 \mathrm{mg} / \mathrm{ml})$, and thrombin $(500$ IU/mg).

\section{Kinetics of in vitro BMP-2 release}

The kinetics of BMP-2 (Cowell Medi Co., Busan, Korea) release from HCF and collagen sponge delivery vehicles (Integra Life Science Co., Plainsboro, NJ) were determined with an enzyme-linked immunosorbent assay (ELISA). Each delivery vehicle containing BMP-2 $(1 \mu \mathrm{g}, \mathrm{n}=8$ per group) was immersed in a 2-ml microcentrifuge tube containing $1.5 \mathrm{ml}$ of PBS, and the tubes were incubated at $37^{\circ} \mathrm{C}$ with continuous agitation. At various time points, the supernatant was collected, and the microcentrifuge tubes were replenished with fresh buffer. The amounts of BMP-2 in the supernatants were determined with an ELISA kit (Human BMP-2 Quantikine ${ }^{\circledR}$; R\&D vehicle, Minneapolis, $\mathrm{MN})$.

\section{Bioactivity of in vitro-released BMP-2}

Calvarial osteoblasts were isolated from calvaria of neonatal (less than 1 day old) Sprague-Dawley rats (SLC, Tokyo, Japan) by a digestive enzymatic process. The bioactivity of BMP-2 released from the delivery systems in vitro was assessed by determining their ability to stimulate alkaline phosphatase (ALP) activity of cultured rat calvarial osteoblasts. Rat calvarial osteoblasts $\left(3 \times 10^{4}\right.$ cells per well) were plated in each well of six-well tissue culture plates (Corning, NY). The HCF and the collagen sponge containing BMP-2 $(1 \mu \mathrm{g})$ were placed on the culture insert (Transwell ${ }^{\mathbb{R}}$; Corning) in the culture plates. The medium was Dulbecco's modified Eagle's medium (Gibco, NY) containing $10 \%(\mathrm{v} / \mathrm{v})$ fetal bovine serum (Gibco) and $1 \%$ penicillin/streptomycin (Pen Strep ${ }^{\mathbb{R}} ;$ Gibco). The medium was changed every 3 days. ALP activity was determined using p-nitrophenol phosphate (Anaspec ${ }^{\circledR}$; San Jose, CA) as the substrate. Rat calvarial osteoblasts were rinsed twice with PBS and lysed in alkaline lysis buffer, followed by three freeze-thaw cycles at $-70^{\circ} \mathrm{C}$ and $37^{\circ} \mathrm{C}$. The aliquots were incubated in glycine buffer containing $2 \mathrm{mg} / \mathrm{ml}$ of p-nitrophenol phosphate. After $30 \mathrm{~min}, 3 \mathrm{~N} \mathrm{NaOH}$ was added to stop the reaction. The absorbance of p-nitrophenol was measured at $405 \mathrm{~nm}$. Total cellular protein was determined using the Bradford reagent (Sigma). The enzyme activity was normalized to the total cellular protein. The experiments were performed in triplicate.

\section{BMP-2 delivery in mouse calvarial defect model}

Six-week-old mice from the Institute of Cancer Research (Orient Bio Co., Gyeonggi-Do, Korea) were anesthetized with xylazine $(20 \mathrm{mg} / \mathrm{kg})$ and ketamine $(100 \mathrm{mg} / \mathrm{kg})$. After shaving the scalp hair, a longitudinal incision was made in the midline of the cranium from the nasal bone to the posterior nuchal line, and the periosteum was elevated to expose the surface of the parietal bones. Using a surgical trephine bur (Ace Surgical Supply Co., Brockton, MA) and a low-speed micromotor, two circular, transosseous defects with a diameter of $4 \mathrm{~mm}$ were produced in the skull. The drilling site was irrigated with saline and bleeding points were electrocauterized. Each animal had two defects, and eight animals were used for each group. The calvarial defects were filled with BMP-2 loaded HCF and collagen sponge. The doses of implanted BMP-2 were $0,0.5,1,2$, and $3 \mu \mathrm{g}$ per defect. The animal study was approved by Institutional Animal Care and Use Committee of Seoul National University (SNU-100203-5).

\section{Bone formation analysis}

Eight weeks after the implantation, the animals were euthanized by $\mathrm{CO}_{2}$ asphyxiation and the skulls were harvested for analysis. Bone formation was evaluated with micro-CT scans ( $n=8$ per group) and histological analysis ( $n$ $=8$ per group). CT images were obtained with a micro-CT scanner (SkyScan-1172; Skyscan, Kontich, Belgium). After the micro-CT examination, the implants were used for histological analysis. All samples were decalcified in $10 \%$ ( $\mathrm{vol} / \mathrm{vol}$ ) formic acid for 7 days, embedded in paraffin, and sectioned transversely at the middle part of the defects at a thickness of $4 \mu \mathrm{m}$. The sections were stained with Goldner's trichrome staining. The percentage of bone formation area in the defect area was calculated as (new bone area / bone defect area) $\times 100$. Bone density was calculated as [new bone area / (new bone area + fibrous tissue area + residual biomaterial area) $\times 100$ (Lee et al., 2010).

\section{Statistical analysis}

Quantitative data were expressed as the mean \pm standard deviation. Statistical analysis was performed with one way analysis of variance (ANOVA) with Tukey's honestly significant difference post-hoc test using SPSS software (SPSS Inc., Chicago, IL). A value of $P<0.05$ was considered statistically significant.

\section{Acknowledgements}

This study was supported by the Samyang Corporation, Daejeon, Korea.

\section{References}

Bessa PC, Casal M, Reis RL. Bone morphogenetic proteins in tissue engineering: the road from laboratory to clinic, part II (BMP delivery). J Tissue Eng Regen Med 2008;2:81-96

Boerckel JD, Kolambkar YM, Dupont KM, Uhrig BA, Phelps EA, Stevens HY, García AJ, Guldberg RE. Effects of protein dose and delivery vehicle on BMP-mediated bone regeneration. Biomaterials 2011;32:5241-51

Carter TG, Brar PS, Tolas A, Beirne OR. Off-label use of recombinant human bone morphogenetic protein-2 (rhBMP-2) for reconstruction of mandibular bone defects in humans. $J$ Oral Maxillofac Surg 2008;66:1417-25

Derner R, Anderson AC. The bone morphogenic protein. Clin 
Podiatr Med Surg 2005;22:607-18

Friedlaender G. Osteogenic protein-1 in treatment of tibial nonunions: current status. Surg technol int 2004;13:249-52

Fu YC, Nie H, Ho ML, Wang CK, Wang CH. Optimized bone regeneration based on sustained release from threedimensional fibrous PLGA/HAp composite scaffolds loaded with BMP-2. Biotechnol Bioeng 2008;99:996-1006

Haidar ZS, Hamdy RC, Tabrizian M. Delivery of recombinant bone morphogenetic proteins for bone regeneration and repair. Part A: current challenges in BMP delivery. Biotechnol Lett 2009;31:1817-24

Issa JP, Bentley MV, lyomasa MM, Sebald W, De Albuquerque RF. Sustained release carriers used to delivery bone morphogenetic proteins in the bone healing process Anat Histol Embryol 2008;37:181-7

Jeon O, Kang SW, Lim HW, Hyung Chung J, Kim BS Long-term and zero-order release of basic fibroblast growth factor from heparin-conjugated poly(I-lactide-co-glycolide) nanospheres and fibrin gel. Biomaterials 2006;27:1598-607

Jeon O, Song SJ, Yang HS, Bhang SH, Kang SW, Sung MA, Lee $\mathrm{JH}$, Kim BS. Long-term delivery enhances in vivo osteogenic efficacy of bone morphogenetic protein-2 compared to short-term delivery. Biochem Biophys Res Commun 2008;369:774-80

Kanakaris NK, Giannoudis PV. Clinical applications of bone morphogenetic proteins: current evidence. J Surg Orthop Adv 2008;17:133-46

La WG, Kang SW, Yang HS, Bhang SH, Lee SH, Park JH, Kim BS. The efficacy of bone morphogenetic protein-2 depends on its mode of delivery. Artif Organs 2010;34: 1150-3

Lee JH, Kim CS, Choi KH, Jung UW, Yun JH, Choi SH, Cho $\mathrm{KS}$. The induction of bone formation in rat calvarial defects and subcutaneous tissues by recombinant human BMP-2, produced in Escherichia coli. Biomaterials 2010;31: 3512-9

Liu HW, Chen CH, Tsai CL, Hsiue GH. Targeted delivery vehicle for juxtacrine signaling growth factor based on rhBMP-2-mediated carrier-protein conjugation. Bone 2006;

\section{9:825-36}

Mont MA, Ragland PS, Biggins B, Friedlaender G, Patel T, Cook S, Etienne G, Shimmin A, Kildey R, Rueger DC. Use of bone morphogenetic proteins for musculoskeletal applications: an overview. J Bone Joint Surg Am 2004;86: 41-55

Patel ZS, Young S, Tabata Y, Jansen JA, Wong MEK, Mikos AG. Dual delivery of an angiogenic and an osteogenic growth factor for bone regeneration in a critical size defect model. Bone 2008;43:931-40

Shields LB, Raque GH, Glassman SD, Campbell M, Vitaz T, Harpring J, Shields CB. Adverse effects associated with high-dose recombinant human bone morphogenetic protein-2 use in anterior cervical spine fusion. Spine 2006;31: 542-7

Takahashi Y, Yamamoto M, Tabata Y. Enhanced osteoinduction by controlled release of bone morphogenetic protein-2 from biodegradable sponge composed of gelatin and $\beta$-tricalcium phosphate. Biomaterials 2005;26:4856-65

Termaat MF, Den Boer FC, Bakker FC, Patka P, Haarman HJ. Bone morphogenetic proteins. Development and clinical efficacy in the treatment of fractures and bone defects. $J$ Bone Joint Surg Am 2005;87:1367-78

Uludag H, D'Augusta D, Palmer R, Timony G, Wozney J. Characterization of rhBMP 2 pharmacokinetics implanted with biomaterial carriers in the rat ectopic model. J Biomed Mater Res 1999;46:193-202

Yang HS, La WG, Bhang SH, Jeon JY, Lee JH, Kim BS. Heparin-conjugated fibrin as an injectable vehicle for sustained delivery of bone morphogenetic protein-2. Tissue Eng Part A 2010;16:1225-33

Yang HS, La WG, Bhang SH, Kim HJ, Im GI, Lee H, Park JH, $\mathrm{Kim}$ BS. Hyaline cartilage regeneration by combined therapy of microfracture and long-term bone morphogenetic protein-2 delivery. Tissue Eng Part A 2011;17:1809-18

Zhao B, Katagiri T, Toyoda H, Takada T, Yanai T, Fukuda T, Chung UI, Koike T, Takaoka K, Kamijo R. Heparin potentiates the in vivo ectopic bone formation induced by bone morphogenetic protein-2. J Biol Chem 2006;281:23246-53 